\title{
Treatment for partial tears of the lateral ligament of the ankle: a prospective trial
}

\author{
S C BROOKS, B T POTTER, J B RAINEY
}

\begin{abstract}
There is debate about the most appropriate form of treatment for partial tears of the lateral ligament of the ankle, which are common after inversion injuries. A prospective trial of four forms of treatment was carried out. The forms of treatment used were: no treatment with only a minimal bandage, Tubigrip support, immobilisation in plaster-of-Paris, and physiotherapy. The end point was taken when the patient returned to work or had a low score on an objective clinical scale. Early mobilisation, with or without physiotherapy, was found to offer the most rapid return to functional activity. Patients who had had their ankle immobilised in plaster-of-Paris required more days off work and longer attendance at a follow-up clinic.

Inversion injuries are common and cause absence from work and discomfort for the patient. These findings suggest that mobilisation with physiotherapy, although not practical for all patients, is the most satisfactory course of treatment.
\end{abstract}

\section{Introduction}

Inversion injuries of the lateral ligament of the ankle are common, especially in young athletic people. The management of these injuries continues to be a subject for discussion and dispute. A prospective trial comparing four different forms of treatment was carried out to resolve some of these problems.

\section{Method}

All patients with inversion injuries seen during a 10-week period at the regional accident unit in Edinburgh were considered for the survey. Patients aged under 12 years or over 65 years were excluded. Informed verbal consent was obtained from the patients who were included. A clinical examination was made in every case followed by anteroposterior and lateral radiographic views. Stress views of the lateral ligament were taken under Entonox analgesia. This provided adequate analgesia and did not delay the patient's departure from the department. All radiographs were reported on by a radiologist. A diagnosis of partial tear of the lateral ligament was made if there was no fracture on the radiograph and no evidence of talar tilt greater than $15^{\circ}$ on the injured side of the stress views taken.

Patients were then allocated at random to one of four groups. The treatment groups were as follows.

Group 1-Twenty-seven patients had no treatment; no support or only a minimal bandage.

Group 2-Twenty-one patients had physiotherapy on the day of presentation or within 48 hours of presentation. This consisted of an iced foot bath, mobilisation, and instruction in normal gait. At the second or third visit "wobble board" exercises were introduced. Treatment was considered complete when the patient could tolerate 10 minutes on the wobble board.

Regional Accident Unit, Royal Infirmary, Edinburgh EH3 9YW S C BROOKS, MRCGP, registrar B T POTTER, MB, CHB, registrar J B RAINEY, MB, CHB, registrar
Group 3-Twenty-eight patients were given double Tubigrip support to wear during daytime and advised to remove it when in bed at night.

Group 4-Twenty-six patients had their ankle completely immobilised in a below-knee plaster-of-Paris cast, weight-bearing being allowed after 48 hours.

If a patient in any group was unable to bear weight on his ankle then crutches were given, but patients were encouraged to bear weight as soon as possible.

Patients were reviewed within 48 hours and then at weekly intervals to assess progress. A check list was prepared to assess the extent of the injury. Five factors were considered-subjective pain, pain on stressing inversion, pain on plantar flexion, swelling, and bruising-and a score of 0 (none) to 3 (severe) was given for each factor. The total was the "clinical score" for each patient; the maximum clinical score was 15 , indicating the severest injury. Patients were assessed on each of four visits. The end point was taken when a patient returned to work or had a clinical score of 0 or 1 .

\section{Results}

A total of 241 patients entered the trial. One hundred and thirtynine were excluded: fractures were shown in $\mathbf{3 0}$ cases and laxity of the lateral ligament was seen in six cases; forty patients opted out of the trial and another 63 cases failed to complete full assessment. This led to a certain skewing of the groups but approximately equal numbers of patients entered each treatment group.

The average initial clinical score for each group is shown in table I. The rate of improvement for each of the treatment groups was then calculated on the clinical score for each visit: these are shown in table II.

TABLE I-Average clinical score of each patient group

\begin{tabular}{|c|c|c|c|c|c|}
\hline Patient group & & & & & $\begin{array}{l}\text { Average } \\
\text { clinical score }\end{array}$ \\
\hline Patients opting out of trial $\quad$. $\quad$. & $\cdots$ & $\cdots$ & . & .. & $6 \cdot 78$ \\
\hline Patients failing to complete assessment & $\cdots$ & $\cdots$ & $\cdots$ & $\ldots$ & 6.96 \\
\hline Patients with fractures . . & .. & . & .. & . & $8 \cdot 60$ \\
\hline Patients with unstable ankles $\ldots$ & .. & . & .. & . & $8 \cdot 33$ \\
\hline Treatment group $1-$ No treatment & . & .. & .. & .. & $6 \cdot 25$ \\
\hline Treatment group 2-Physiotherapy .. & $\cdots$ & $\cdots$ & $\cdots$ & $\because$ & $6 \cdot 50$ \\
\hline Treatment group 3-Tubigrip & . & .. & .. & .. & $7 \cdot 03$ \\
\hline Treatment group 4-Plaster-of-Paris & . & .. & .. & .. & $7 \cdot 20$ \\
\hline
\end{tabular}

TABLE II-Average clinical score for each visit for the different treatment groups

\begin{tabular}{lccccc}
\hline & First visit & Second visit & Third visit & Fourth visit & Fifth visit \\
Group 1 & 6.2 & 2.8 & 1.0 & 2.0 & 0.2 \\
Group 2 & 6.5 & 4.9 & 1.2 & 0.4 & 0.5 \\
Group 3 & 7.1 & 5.1 & 1.0 & 0.5 & 1.0 \\
Group 4 & 7.2 & 6.2 & 1.6 & 0.8 & \\
\hline
\end{tabular}

The financial cost of these injuries may be estimated from the number of days off work that the patients required. The average number of days off work and average number of days spent at the follow-up clinic were assessed for each group. In group 1 the numbers were 5.1 and 23.6 respectively; in group $2,6.0$ and 21.4 ; in group 3 , $7 \cdot 5$ and $21 \cdot 5$, and in group $4,14 \cdot 0$ and $25 \cdot 0$.

An indication of the severity of the initial injury was taken from the number of patients using crutches. Patients in group 4 were all given crutches for the first 48 hours to ensure full drying of their plaster-ofParis. Only seven patients in group 1 , four in group 2, and three in group 3 used crutches. The crutches in groups 1 and 3 were used for a maximum of three days. Patients receiving physiotherapy all left their crutches after the first session of treatment. 
Patients were asked for their comments on the treatment they received. In the group receiving no treatment 15 of the 27 patients would have preferred some sort of support to the ankle and felt that their treatment was unsatisfactory. Despite their subjective comments they in fact had the shortest time off work. This may well reflect the less serious nature of the injury in this group as they had the best initial clinical score. All the patients who received physiotherapy were impressed by the treatment. They could all walk away from the department and thought the treatment greatly aided mobility and reduced pain and swelling. Patients who were given Tubigrip support had few comments, and only one patient would have preferred crutches. All patients who had had their ankle immobilised in plasterof-Paris thought they had good pain relief but they were off work longer and had a longer attendance at the follow-up clinic. They complained of stiffness on removal of the plaster-of-Paris and found it more difficult to regain full mobility.

\section{Discussion}

The comparative efficiency of the different forms of treatment available in inversion injuries of the ankle is difficult to demonstrate. This is due to a combination of factors; difficulty in assessing the degree of injury or tear in the lateral ligament and the essential time-limited nature of the disability are among the most important.

These reasons also lead to poor patient co-operation in any trial to find the appropriate treatment. In our trial just over 100 patients either opted out of the trial or failed to attend for complete assessment. Previous reports indicate that patients returned to normal functional activities after an average of 13 days. ${ }^{1}$ Our report indicates a return to work in an average of eight days. There have been series comparing the results of differing treatments ${ }^{2}{ }^{3}$ but these were mainly concerned with severe injury. The degree of talar tilt that is believed to indicate laxity of the ankle leading to instability is still controversial. In one series ${ }^{4}$ there was a sharp cut-off at $13^{\circ}$ of talar tilt in normal ankles and in sprained ankles a similar cut-off was seen. Allowing for $1^{\circ}$ or $2^{\circ}$ accuracy this set our limit of talar tilt at $11^{\circ}$ to $15^{\circ}$; we therefore took $15^{\circ}$ as our abnormal limit.

It is estimated that there is about one inversion injury per 10000 persons per day. ${ }^{5}$ Investigation and treatment can be expensive. While not all patients can be expected to be treated with physiotherapy, this study shows that mobilisation, with early physiotherapy or even without, offers the most rapid return to functional activity.

\section{Requests for reprints to: Dr B T Potter.}

We wish to thank the staff of the regional accident unit for help in this work, Dr K Little for permission to undertake the study and support in its preparation, Miss Henderson, physiotherapy department, and Caroline McKenzie for typing it.

\section{References}

1 Dameron T. Management of acute ankle sprains. South Med 7 1977;70: 1166-7.

2 Freeman MAR. Treatment of ruptures of the lateral ligament of the ankle. f Bone foint Surg 1965;47B:661-8.

${ }^{3}$ MacCartee CC. Taping treatment of severe inversion sprains of the ankle. Am 7 Sports Med 1977;5:246-7.

4 Rubin G, Witten M. The talar tilt angle and the fibular collateral ligaments of the ankle. $\mathcal{F}$ Bone Foint Surg 1960;42A:311-25.

5 Ruth CJ. Surgical treatment of injuries of the collateral ligaments of the ankle. F Bone foint Surg 1961 ;43A :229-39.

(Accepted 3 December 1980) tinuing pain or those who clinically have a fracture requiring immobilisation. This would produce a large saving in NHS resources.

\section{Introduction}

Inversion injuries of the ankle are among the commonest injuries presenting to accident and emergency departments. General practitioners refer patients to the department for $x$-ray examination to exclude injury to the bone. Radiographic examination of all these cases represents a considerable drain on limited resources : ankle radiographs account for about $2 \%$ of all radiological examinations. A prospective study was carried out to determine the accuracy of clinical examination of the ankle joint after inversion injury. Radiographic examination was made in every case, and stress views were taken to exclude increased laxity of the ankle joint. This was compared with the clinical assessment recorded before the $\boldsymbol{x}$-ray film was taken or viewed by the clinical assessor.

\section{Method}

All patients with inversion injuries seen during a 10-week period at the regional accident unit in Edinburgh were included in the survey,
S C BROOKS, MRCGP, registrar

B T POTTER, MB, CHB, registrar

J B RAINEY, MB, CHB, registrar 